\title{
Quasi-Active Thermography for Structural Health Assessment of Large Geomembranes
}

\author{
Yue Ma ${ }^{1, a,{ }^{*}}$, Leslie Wong ${ }^{1, b}$, Benjamin Steven Vien ${ }^{1, c}$, Thomas Kuen ${ }^{2, d}$ \\ Nik Rajic ${ }^{3, \mathrm{e}}$, L.R. Francis Rose ${ }^{3, \mathrm{f}}$, Jayantha Kodikara ${ }^{4, g}$, and Wing Kong Chiu 1, h \\ ${ }^{1}$ Department of Mechanical \& Aerospace Engineering, Monash University, Clayton, VIC 3008, \\ Australia \\ ${ }^{2}$ Melbourne Water Corporation, 990 La Trobe Street, Docklands, VIC 3008, Australia \\ ${ }^{3}$ Defence Science and Technology Group, 506 Lorimer Street, Fishermans Bend, VIC 3207, \\ Australia \\ ${ }^{4}$ Department of Civil Engineering, Monash University, Clayton, VIC 3008, Australia \\ ayue.ma@monash.edu, bLeslie.Wong@monash.edu, cben.vien@monash.edu, \\ dThomas.Kuen@melbournewater.com.au, eNik.Rajic@dst.defence.gov.au, \\ fFrancis.Rose@dst.defence.gov.au, 9Jayantha.kodikara@monash.edu, \\ hwing.kong.chiu@monash.edu
}

Keywords: Quasi-Active Thermography, Structural Health Monitoring, Geomembrane, Floating Covers, Sewage Treatment Plant

\begin{abstract}
High density polyethylene (HDPE) geomembranes (approximately 8 hectares each) are employed as floating covers at the wastewater treatment plant of Melbourne Water in Werribee, Australia. The anaerobic lagoons at the plant rely on these HDPE geomembrane floating covers to capture both the biogas and odours. Given the nature of the plant and the harsh environmental conditions, a non-contact inspection method that can cover a vast expanse is the preferred approach for the structural health monitoring and assessment of the cover. This paper presents an exploratory investigation on the use of a quasi-active thermography technique to detect the presence of artificially induced part-through defects on a HDPE geomembrane specimen. The proposed method utilises a naturally occurring heat source (solar radiation) as the thermal stimulus. An infrared thermal camera and a pyranometer were used to record the thermal responses of the HDPE material as a result of solar intensity variation. The viability of using periodic cloud cover transients to drive this inspection technique is reported. In addition, an image processing algorithm is formulated based on the relative summation of the transient events to enhance the identification of the defects. The findings show that the observed thermal transients can be used to define the presence of defects both when the underside of the material is in contact with water or with air, and thereby provides a promising approach for the structural health monitoring of these high-value assets.
\end{abstract}

\section{Introduction}

Geomembranes have been widely used in both environmental and mining applications, particularly for landfill liners, as well as floating covers in anaerobic and aerobic reactors in wastewater treatment plants. Raw sewage enters the large covered lagoons at the start of the wastewater treatment process, and biogas is a by-product of the anaerobic digestion process that is the first step in breaking down the untreated sewage. The HDPE membrane covers which float on the sewage in these lagoons play a significant role, (1) in harnessing the biogas for the generation of electricity that powers the entire plant and (2) preventing the odourous and 
greenhouse gases from being released to the atmosphere. There are several large floating covers located at Melbourne Water's Western Treatment Plant (WTP) at Werribee, Melbourne, Australia. These floating covers are made out of $2 \mathrm{~mm}$ thick high-density polyethylene (HDPE) material and cover an area of $470 \mathrm{~m} \times 170 \mathrm{~m}$ (refer to Figure 1). Approximately $65,000 \mathrm{~m}^{3}$ of biogas per day is harvested from these covers, which is used to generate $7 \mathrm{MW}$ of renewable energy per day [1].

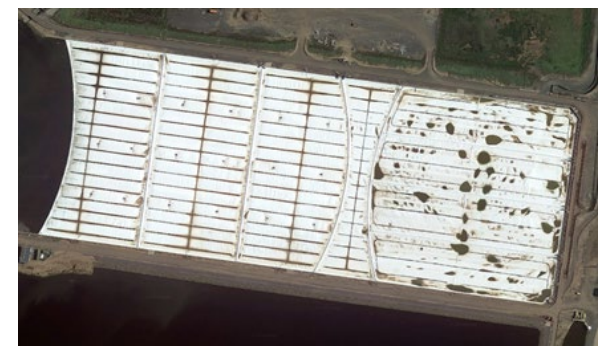

Figure 1. Aerial view of floating cover in western plant treatment

Since the raw sewage is untreated when entering the lagoon system, fats, oils, solids or other fibrous materials may eventually be transported to the surface of the lagoon under the cover[2]. This material, known as scum, can expand in spread and depth and become harder over time, potentially developing into a large solid mass that is referred to as a "scumberg" as parts of it may rise above the water surface level even when under the cover. The existence of scumbergs results in the local deformation of the cover, and furthermore, hardened scum may scratch the underside of the geomembrane if it moves and generate part-through defects. These floating covers are valuable assets for the utilities and hence developing an effective structural health monitoring method to evaluate the integrity of these large floating covers is necessary for safe, effective, and efficient operation of the plant. The current evaluation method requires an inspector to walk on these covers and conduct a time-consuming visual inspection. Therefore, a non-contact structural health monitoring (SHM) technology can potentially improve the efficiency and safety of the inspection of these large-scale covers.

This paper introduces a quasi-active thermography technique to detect the presence of partthrough defects on the HDPE cover material. The results reported were obtained from a series of experiments conducted on a rooftop that is fully exposed to the prevailing weather conditions. The proposed technique utilises variations in the ambient solar radiation to detect and identify these defects. This approach provides a new quasi-active thermal imaging method to evaluate the structural integrity of large-scale floating structures, as the basis for SHM.

\section{Quasi-active thermography}

Thermography, or the use of thermograms, is based on the infrared energy emitted by an object and detected with a thermal camera. Thermographic methods can be classified as either active or passive. Active methods require a thermal stimulus from a power source external to the object. The most commonly used source is an optical lamp which thermally stimulates the surface with either a relatively short heat pulse or a periodic fluctuation of heat. Defects are identified by temperature contrasts arising from differential rates of heating and cooling relative to undamaged regions of the object, which are caused by differences in thermal conductivity or thermal mass. These temperature contrasts can be detected as a variation in infrared (IR) emission from the surface [3]. 
Omar [3] applied active thermography to evaluate the bond strength and adhesion integrity of a HDPE plastic joint, demonstrating that defects within HDPE can be monitored. Flores-Bolarin [4] applied active thermography to determine the depth of defects in HDPE subjects .

In passive thermography, monitored objects have naturally occurring thermal contrasts which are established via ambient or operational stimuli - e.g. boiler operation [5], and therefore do not require a separate heat source. Both methods have been used to monitor a wide range of structures [6-8], and polymer materials [9, 10].

The size of the floating cover poses several challenges to the application of active thermography. One of them is the requirement to heat the entire structure simultaneously and uniformly. In addition, the use of electronic devices in proximity to the covers is restricted because of the potentially flammable environment associated with the methane-rich biogas produced under the covers. The application of conventional active thermography on structures as large as the floating covers at WTP would require a large amount of thermal energy and time to ensure the uniform flow of the heat flux into the structure and is therefore considered impracticable for use in the real-world of the WTP.

In this paper, a quasi-active thermography method is deployed[11] where natural sunlight is proposed as the heat source for conducting an inspection of these large structures. The radiation from sunlight is considered to be relatively uniformly deposited over the expanse of the covers at WTP. The shading from clouds will temporarily block the solar radiation and will generate a thermal transient on the surface of the geomembrane material. This paper investigates whether variations in cloud-cover can provide transients in timescales that enable the detection of defects in the cover material. The aim of this study is to experimentally investigate the application of quasi-active thermography under laboratory conditions on an exposed rooftop.

In this study, a sample of the HDPE geomembrane material in use at WTP, with artificially induced defects, was supported in a test rig on an exposed roof top. The test membrane had a series of part-through thickness reductions simulating defects. Given that the floating cover can be in direct contact with the raw sewage, and can be separated from the raw sewage by the presence of the biogas, the test membrane was supported on the test rig with parts of the underside of the cover material in contact with water, and the remaining parts in contact with an air gap between the surface of the water and the membrane (to simulate the trapped biogas).

\section{Identification of transient event due to cloud movement}

The IR emissivity spectrum of HDPE geomembrane was measured using Fourier-Transform Infrared (FTIR) spectroscopy, which indicated that the HDPE geomembrane is suitable for infrared thermography. The HDPE geomembrane specimen (1 m $\times 1 \mathrm{~m}$ x $2 \mathrm{~mm}$ thickness $)$ was clamped onto the aluminium test rig, as shown in Figure 2(a). An Apogee SP-110 pyranometer [12] was installed on the specimen to monitor the irradiation of the specimen surface. An A615 FLIR infrared thermal camera [13] which contains an uncooled Vanadium Oxide detector array of size $640 \times 480$, was placed beside the geomembrane to record the temperature of the surface of the geomembrane. This equipment setup allows continuous monitoring of the power of solar radiation, through which the transient events due to cloud interference (i.e. shading) can be identified and then correlated with the information obtained from infrared imaging over an extended period of time. The infrared imaging during these transient events is studied in detail. A preliminary test was first conducted by placing the experimental set up outdoors for 1 hour (see Figure 2(b)) in order to identify the transient event and optimise the resolution and time recording parameters. Both solar intensity and thermal imagery was recorded at a sampling rate of $3 \mathrm{~Hz}$ over the test period. Figure 3 shows the solar intensity history measured by the 
pyranometer, as well as the temperature variation measured at a point from the thermal imagery, over an observation window of 1 hour. The experiment was conducted on a cloudy day. The pyranometer data showed that the sun was unobstructed by clouds on three occasions during the test period (see Figure 3). During each cloud transition event, associated temperature transients in the membrane are evident. The first event which happened between 12:12:30 and 12:23:20 of the experiment is labelled in Figure 3. The transient event started at 12:12:30 when the pyranometer recorded a change in solar intensity. The temperature transient in the membrane is evident after a few seconds into the cloud-cover event, i.e. the geomembrane temperature increased. According to the pyranometer data, the sun reappeared from the cloud at 12:23:20. A corresponding thermal transient end point was recorded after several seconds into this event (geomembrane temperature increased). Figure 3 suggests that a cloud-cover event can result in a temperature change in the membrane of up to $5^{\circ} \mathrm{C}$.

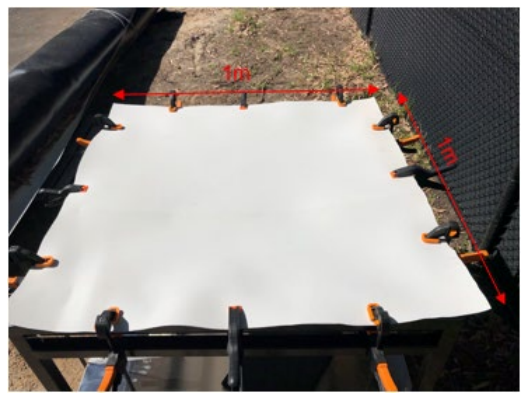

(a)

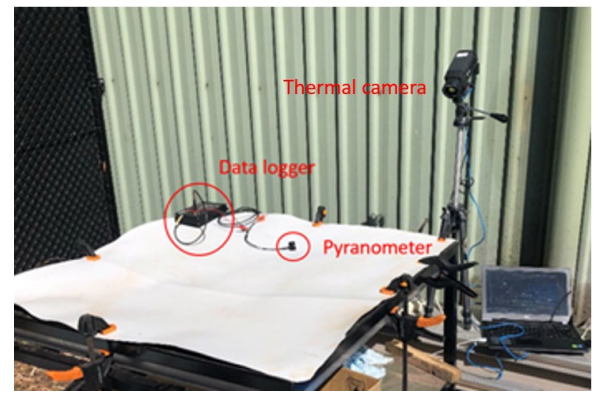

(b)

Figure 2. (a) HDPE geomembrane specimen (b) Experiment set up of quasi-active thermal imaging

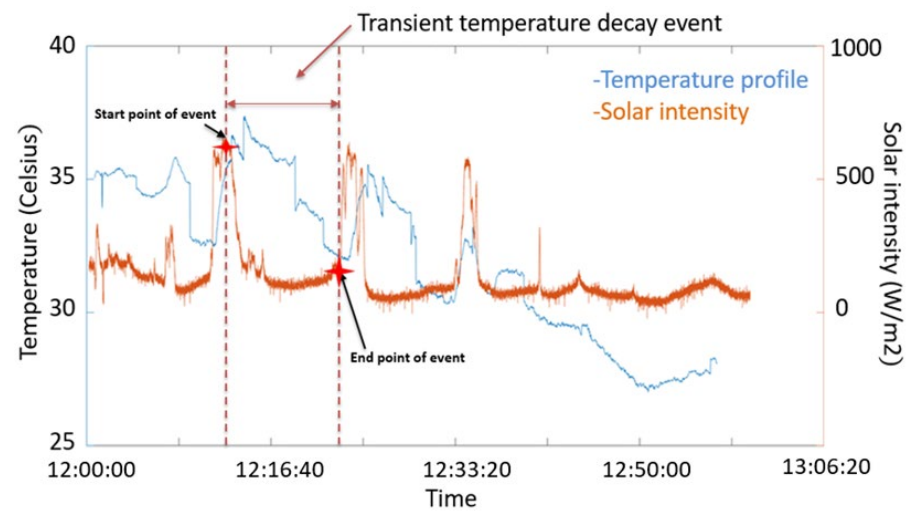

Figure 3. Temperature profile on HDPE membrane and solar intensity measured in-site

\section{Processing of thermal image}

In the above validation investigation, it was shown that the thermal transients of the HDPE geomembrane can be generated by exposure to cloud-cover events. The quality of the raw thermal images in thermography is inevitably degraded due to the background temperature variations at different times in a day, making it difficult to set an appropriate threshold of temperature variation that would indicate the presence of a defect, and the defects are difficult to identify in a single captured thermal image. Therefore, the proposed technique compares the relative temperature changes for a series of thermal images that are recorded at different times during the day. A defect detection algorithm is developed to relate the measured thermal signal (i.e. from the camera) with the radiation signal (i.e. from the pyranometer), and to enhance the 
ability to detect the part-through defects on the floating cover. The hypothesis for the algorithm is as follows: clouds will at first provide shade thus reducing solar irradiation of the cover material, thereby initiating a transient event; hence, this will lead to a change in temperature on the surface of the geomembrane. So, to enhance the quality of the thermal image, the following temperature change index $\Delta T$ is formulated,

$$
\Delta T=\sum_{j=1}^{m} \sum_{i=1}^{n_{j}} T_{j(i)}(x, y)-T_{j(1)}(x, y) \quad(\text { Eqn. } 1)
$$

The index $\mathrm{i}$ represents the number of the currently recorded frame in each event, the index $\mathrm{j}$ is the number of cloud shading events in the experiment, $m$ is the total number of transient events, and $\mathrm{n}_{\mathrm{j}}$ is the total number of frames in the jth transient event. $T_{j(i)}(x, y)$ is the temperature in frame $\mathrm{i}$ in event $\mathrm{j}$ at pixel $(\mathrm{x}, \mathrm{y})$ and $T_{j(1)}(x, y)$ is the temperature in the initial frame in event $\mathrm{j}$ at pixel $(\mathrm{x}, \mathrm{y})$. It is anticipated and assumed that multiple cloud shading events will occur over the duration of an experiment. This index calculates the sum of temperature changes at every pixel in the infrared image by comparing with the first frame in the corresponding event as defined by the trigger. A threshold signal value in the pyranometer data is used as a trigger to acquire the transients. Transient events are manually selected based on the local maximum solar intensity and local minimum solar intensity. The cooling transient event is set when a cloud shading is detected (corresponding to a decrease in solar intensity).

\section{Detection of part-through defects when touching water}

At the wastewater treatment plant, it is known that the geomembrane can both be in contact with the liquid sewage underneath and raised from the sewage by pockets of biogas that lift up the cover in other regions. Assuming that defects can be present in regions that are both in contact with liquid sewage or with biogas, this experimental investigation focuses on identifying defects when the underside of the HDPE sample is both on air and on water.

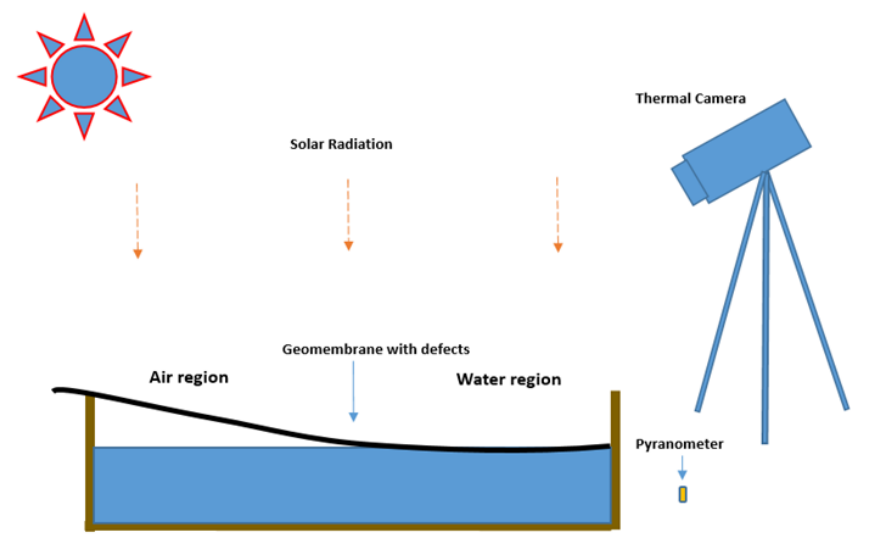

Figure 4. Experiment set up of detecting part-through defects on air and on water

As shown in Figure 4, a part of the membrane specimen floats on the water to simulate the liquid sewage region at the treatment plant and the remaining portion has an air gap to simulate the pockets of biogas beneath the floating cover. As shown in Figure 5, a series of part-through defects were created on the underside surface of a $0.5 \mathrm{~m} \times 1 \mathrm{~m} \times 2 \mathrm{~mm}$ membrane using laser cutting equipment. The dimensions of these defects are shown in Table 1. Defects number 1, 2, 5 and 6 were located in the air region, and defects number 3, 4, 7 and 8 were located in the water region. The experiment was taken outside for 1 hour and exposed to multiple cloud shading 
events. Figure 6 shows solar intensity in these regions decreases abruptly and results in thermal transients in the membrane material. As shown in Figure 7 (a), the regions of air and water contact are readily distinguished in the raw thermal image. The higher thermal resistance of air compared with water resulted in a local temperature increase relative to the part of the membrane resting on the water surface. The right section of the test specimen is located over the water region. The heat absorbed in the membrane in this region is transferred efficiently into the water which has a higher specific heat than air, resulting in a lower temperature relative to the left section. Using the raw thermal image only defect number 1 and 2 which have the largest depth and width can be identified, whereas the defects in the water region could not be readily detected.

The temperature variations in thermal imagery corresponding to the transient events were processed using Eqn 1. The results obtained are shown in Figure 7 (b). It can be seen that all the defects can now be clearly identified, and the profiles of defects in the air region are clearer. Defects in the water region can be detected, but due to heat being more easily absorbed by water, the profiles are not as clear as those in the air region. In addition, the profiles of defects 5-8 which have $0.5 \mathrm{~mm}$ defect depth are not as clear as the profile of defects 1-4 which have $1 \mathrm{~mm}$ defect thickness.

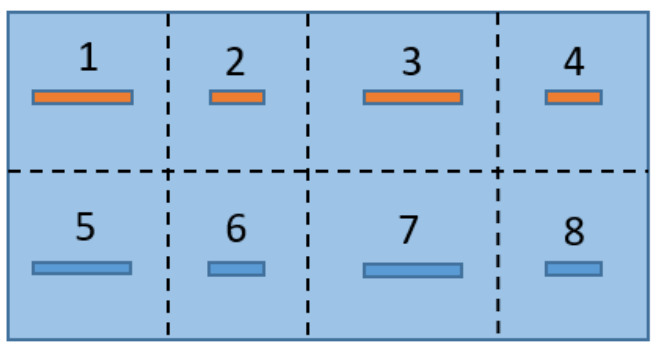

Figure 5. HDPE geo-membrane with different dimensions of under surface scratch damage

Table 1 Details of artificially created damages in each region on the membrane

\begin{tabular}{cccc}
\hline Defects number & $\begin{array}{c}\text { Defect length } \\
(\mathrm{cm})\end{array}$ & $\begin{array}{c}\text { Defect width } \\
(\mathrm{cm})\end{array}$ & $\begin{array}{c}\text { Defect thickness } \\
(\mathrm{mm})\end{array}$ \\
\hline 1 & 10 & 1 & 1 \\
2 & 5 & 1 & 1 \\
3 & 10 & 0.5 & 1 \\
4 & 5 & 0.5 & 1 \\
5 & 10 & 1 & 0.5 \\
6 & 5 & 1 & 0.5 \\
7 & 10 & 0.5 & 0.5 \\
8 & 5 & 0.5 & 0.5 \\
\hline
\end{tabular}

These results demonstrate that thermography based on naturally occurring transient events can be effectively exploited to capture part-through defects of HDPE materials in contact with air or water. It is also noted that defects on a membrane with an underside in contact with water are harder to detect than those on regions with an underside in contact with air. The results in Figure 7 (b) show that detectability is greatly enhanced by calculating the index presented in Eqn. 1 for defects in contact with air (simulating biogas) or water (simulating liquid sewage). 


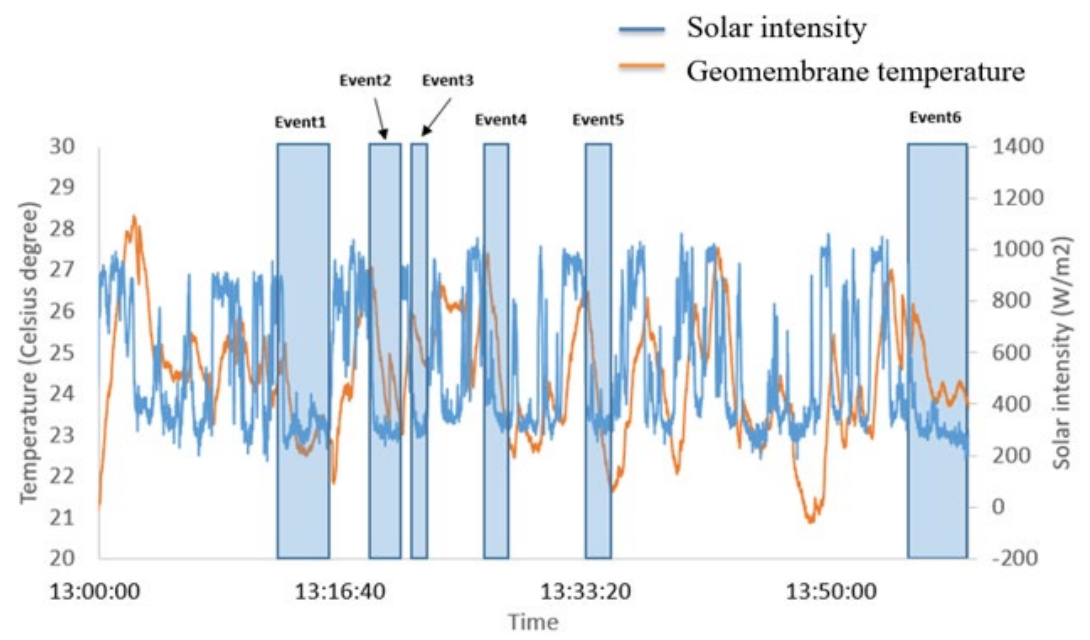

Figure 6. History of temperature evolution and solar intensity with 6 highlighted transient events

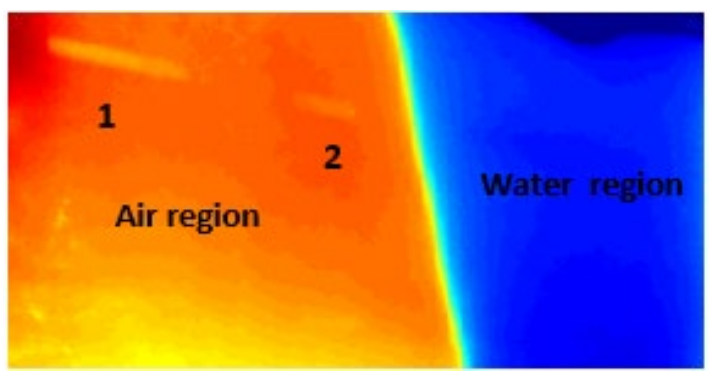

(a)

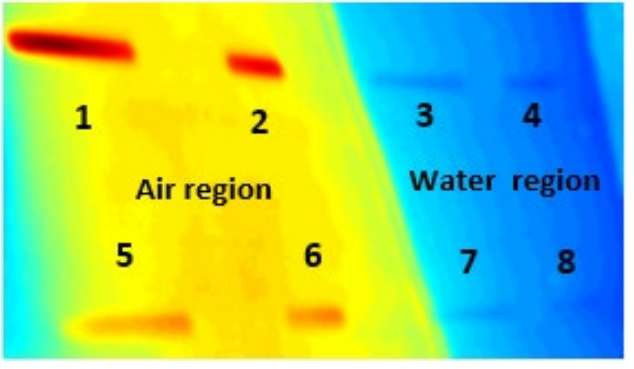

(b)

Figure 7. (a) Raw infrared thermal image (b) processed thermal image with Eqn (1

\section{Conclusions}

This paper reports a quasi-active thermography technique to evaluate the structural health condition of large-scale floating covers in sewage treatment plants. The technique relies on transient temperature decay events caused by variations in solar radiation on the surface of HDPE geomembranes due to variations in cloud cover. Thermal imaging is then possible for defects that induce variations in thermal conductivity or thermal mass of the membrane. An algorithm was devised to aid defect detection using sunlight with transient cloud shading events. From the above experiments, the results and findings indicate that:

- Quasi-active thermography with the aid of naturally occurring solar radiation fluctuations is able to detect part-through defects on the cover. Transient events from cloud cover were suitable for thermal imaging of defects on HDPE geomembranes.

- Thermography can distinguish between membrane regions that are in contact with air relative to water, and part-through defects of different depths in these two regions can be imaged. Defects in air contact regions are easier to detect.

In future work, the quantitative relation between defect thickness and temperature evolution in the material will be investigated, and in-situ verifications carried out. The technologies developed have the potential to provide an economical, real-time and robust method to maintain the structural integrity of large-scale membrane-like structures and extend the service life span of geomembranes in treatment plants, thereby providing a promising approach for SHM of these high-value assets. 


\section{Acknowledgement}

The financial support provided by Melbourne Water and Australia Research Council Linkage Grant (ARC LP170100108) is gratefully acknowledged.

\section{References}

[1] Chiu, W.K., et al., Large Structures Monitoring Using Unmanned Aerial Vehicles. 2017. p. 415-423. https://doi.org/10.1016/j.proeng.2017.04.503

[2] Wong, L., et al., Remote Monitoring of Floating Covers Using UAV Photogrammetry. Remote Sensing, 2020. 12(7): p. 1118. https://doi.org/10.3390/rs12071118

[3] Omar, M., et al., Infrared thermography for inspecting the adhesion integrity of plastic welded joints. NDT \& E International, 2006. 39(1): p. 1-7. https://doi.org/10.1016/j.ndteint.2005.04.008

[4] Flores-Bolarin, J. and R. Royo-Pastor. Infrared thermography: A good tool for nondestructive testing of plastic materials. in Proceedings of the 5th European Thermal-Sciences Conference, Eindhoven, The Netherlands. 2008.

[5] Taole, R., R. Falcon, and S. Bada, The impact of coal quality on the efficiency of a spreader stoker boiler. Journal of the Southern African Institute of Mining and Metallurgy, 2015. 115(12): p. 1159-1165. https://doi.org/10.17159/2411-9717/2015/V115n12a3

[6] Swiderski, W. and D. Szabra. Possibility of defect detection in multi-layered composite materials used for military applications by IR thermography. in Proc. of the 5th International Workshop on Advances in Signal Processing for Non-Destructive Evaluation of Materials, Quebec City, Canada. 2005.

[7] Jorge Aldave, I., et al., Review of thermal imaging systems in composite defect detection. Infrared Physics and Technology, 2013. 61(C): p. 167-175.

https://doi.org/10.1016/j.infrared.2013.07.009

[8] Rajic, N. and S. Galea, Thermoelastic stress analysis and structural health monitoring: an emerging nexus. Structural Health Monitoring, 2015. 14(1): p. 57-72.

https://doi.org/10.1177/1475921714548936

[9] Steinberger, R., et al., Infrared thermographic techniques for non-destructive damage characterization of carbon fibre reinforced polymers during tensile fatigue testing. International Journal of Fatigue, 2006. 28(10): p. 1340-1347. https://doi.org/10.1016/j.ijfatigue.2006.02.036 [10] Kafieh, R., T. Lotfi, and R. Amirfattahi, Automatic detection of defects on polyethylene pipe welding using thermal infrared imaging. Infrared Physics \& Technology, 2011. 54(4): p. 317325. https://doi.org/10.1016/j.infrared.2010.12.010

[11] Ma, Y., et al., Quasi-Active Thermal Imaging of Large Floating Covers Using Ambient Solar Energy. Remote Sensing, 2020. 12(20): p. 19. https://doi.org/10.3390/rs12203455

[12] Apogee, Apogee instruments owner's manual pyranometer models SP-110 and SP-230, Logan.USA, Editor. 2020.

[13]FLIR, User's manual FLIR A6xx series, O. FLIR systems: Wilsonville, United States Editor. 2016. 